Norka Machicao Chacón 1,a Juan F. Hernández Añaños 1,2,b

Facultad de Estomatología Roberto Beltrán, Universidad Peruana Cayetano Heredia. Lima, Perú.

2 Sociedad Peruana de Endodoncia. Lima, Perú.

a Residente de Endodoncia.

b Magíster y Especialista en Endodoncia.

\section{Correspondencia:}

Norka Machicao Chacón

Av. Godoy 888 SMP

Lima, Perú

Teléfono: 51-962354698

E-mail: norka.machicao@upch.pe

\title{
Número de sesiones para realizar tratamiento de conductos en piezas permanentes.
}

Machicao $N^{1, a}$, Hernández $J F^{1,2, b}$. Número de sesiones para realizar tratamiento de conductos en piezas permanentes. Rev Estomatol Herediana. 2013 Ene-Mar;23(1):11-7.

\section{RESUMEN}

El propósito del presente estudio fue determinar el número de sesiones utilizadas para realizar tratamientos de conductos en piezas permanentes tratadas en el Servicio de Endodoncia - Posgrado y Pregrado de la Facultad de Estomatología de la Universidad Peruana Cayetano Heredia (FE-UPCH) en el año 2010. Se encontró que en Pregrado es igual de frecuente hacer tratamientos de conductos en una o en dos sesiones (44,25\% para cada uno de ellos), mientras que en Posgrado lo más frecuente es realizar tratamientos en una sesión.

Palabras clave: TRATAMIENTO ENDODÓNTICO, MÚLTIPLES SESIONES, UNA SESIÓN.

Number of sessions to perform root canals in permanent teeth.

\section{ABSTRACT}

The purpose of this study was to determine the number of sessions used to perform root canals on permanent teeth treated in the Department of Endodontics of the Postgraduate and Undergraduate in the Faculty of Dentistry of the Universidad Peruana Cayetano Heredia in 2010. It was found that in the Undergraduate is common finish root canals treatments in one or two sessions (44.25\% each), while for the Postgraduate Endodontics is frequently to perform root canals in one session.

Key words: TREATMENT ROOT CANAL, MULTIPLE SESSIONS, ONE SESSION.

\section{Introducción}

El tratamiento endodóntico tiene como objetivo la eliminación del tejido pulpar y microorganismos, de manera que se pueda lograr una adecuada conformación de los conductos radiculares tratados a fin de permitir una obturación con un sellado hermético y tridimensional de los mismos.

En la actualidad, existen dos corrientes de opinión para realizar dicho tratamiento: en una sesión o en múltiples sesiones. La primera de ellas se encuentra representada principalmente por autores norteamericanos, quienes resaltan dicha corriente como "mecánica", defendiendo la posición que el tratamiento de conductos en los que se realice un adecuada preparación biomecánica, correcta irrigación y ausencia de complicaciones no pre- sentaría contraindicaciones de ser concluido en una misma sesión (1).

El debate surge en los casos con pulpa necrótica e indicios de lesión apical. Si es que se debe o no realizar en una sesión; ya que habría ocurrido propagación y proliferación bacteriana en todo el sistema de conductos radiculares, la que sería imposible de eliminar $(2,3)$. Es por ello, que en estos casos, aconsejan utilizar una terapia de varias sesiones con medicación intraconducto. Esta escuela es llamada "biológica" y es representada en su mayoría por autores europeos y latinoamericanos, quienes muestran consideración a estructuras periapicales y su proceso de reparación. Cabe resaltar que cada una de estas corrientes no presentan principios excluyentes entre sí $(1,4,5)$.
Es necesario considerar que se pueden emplear diferentes procedimientos terapeúticos, según la condición biológica de la pieza dentaria a tratar, su estado patológico, la habilidad del odontólogo, la disponibilidad de instrumental y las preferencias del paciente $(6,7)$.

Las indicaciones a tener en cuenta en los tratamientos de conductos realizados en una sesión incluyen: dientes vitales con anatomía no complicada, pacientes medicados con corticoides $\mathrm{o}$ en tratamiento con anticoagulantes, pacientes aprehensivos, que requieran sedación o anestesia general y aquellos que presenten hemofilia o trastornos mentales $(8,9)$.

Algunas contraindicaciones de la terapia endodóntica en una sesión son: pieza necrótica con sintomato- 
logía y sin trayecto sinusal para el drenaje, piezas con anatomía compleja, niños que no estén adaptados a terapias odontológicas, la mayoría de retratamientos, en casos de periodontitis apical aguda con dolor severo a la percusión y en pacientes con problemas de articulación temporomandibular $(8,9)$.

Las ventajas de realizar el tratamiento de conductos en una sesión son: reducir a una el número de sesiones a las que el paciente tiene que asistir para el tratamiento endodóntico posibilitando la rehabilitación en menos tiempo (reduciendo probabilidades de microfiltración coronaria), además de reducir la ansiedad del paciente. Para el operador es una ventaja debido a que está familiarizado con la anatomía interna y la posición de los conductos, $\mathrm{y}$ se reduce costo y tiempo $(8,9)$.

Las desventajas del tratamiento endodóntico en una sesión son: al prolongarse la duración del tratamiento puede ocasionar aumento de la fatiga del paciente reduciendo su cooperación, el manejo de las agudizaciones se dificulta debido a la presencia del conducto obturado (especialmente en el tercio apical), se necesita experiencia y habilidad para realizar tratamientos en una sesión, no cabe la posibilidad de administrar medicamentos en el conducto y se produce mayor cansancio del operador a causa de la necesidad de concentración en la ejecución del tratamiento $(8,9)$.

La evolución de las radiografías convencionales que han dado paso a las digitales, el uso de tomografías, localizadores apicales, instrumentos rotatorios de $\mathrm{NiTi}$, los protocolos de irrigación y la obturación termoplástica han hecho posible que muchos de los casos indicados para el tratamiento de conductos puedan ser completados en una sola sesión $(3,10)$, cumpliendo con el objetivo de eliminar la microflora existente en el conducto radicular. Aunque esto no puede conseguirse de forma fiable sin administrar medicamentos dentro del conducto $(8,11,12)$ e incluso, aún con ello su reducción no es $100 \%$ efectiva, debido a que existen zonas inaccesibles a la instrumentación $(2,13)$.

La presente investigación tuvo como objetivo determinar el número de sesiones para realizar tratamientos de conductos en piezas permanentes tratadas en el Servicio de Endodoncia - Posgrado y Pregrado de la Facultad de Estomatología de la Universidad Peruana Cayetano Heredia (FE-UPCH) en el año 2010.

\section{Material y Métodos}

Este estudio fue de tipo descriptivo, retrospectivo, observacional y transversal.

La muestra del estudio estuvo conformada por 305 tratamientos de conductos registrados en las historias clínicas (174 en pregrado y 131 en posgrado), determinado con un nivel de confianza de $95 \%$.

Los criterios de exclusión fueron: tratamientos de conductos de piezas permanentes con diagnóstico de ápice abierto, anatomía radicular compleja (dens in dente, conductos calcificados, dilaceraciones severas), tratamientos de conductos incompletos o piezas dentarias cuyos conductos no hayan sido obturados en su totalidad, piezas anteriores con más de un conducto radicular y tratamientos que no cuenten con la firma del docente encargado.

Los criterios de inclusión fueron: tratamientos de conducto de piezas permanentes iniciadas y concluidas en el Servicio de Endodoncia de Posgrado y Pregrado de la FEUPCH en el año 2010. Considerando datos de las hojas de evolución, siendo complementadas a su vez por las hojas de evaluación.

Se realizó un análisis de las variables: número de sesiones de trabajo, diagnóstico pulpar y periapical. Los resultados se presentan en tablas de frecuencia acumulada, mostrando la distribución porcentual de las variables mencionadas considerando el número de sesiones de trabajo.

La distribución de la frecuencia se realizó relacionando los siguientes datos: número de sesiones para culminar un tratamiento endodóntico, número de sesiones vs diagnóstico pulpar, número de sesiones vs diagnóstico periapical.

\section{Resultados}

Al inicio del proyecto se consideró dividir la población de estudio en dos grupos: pregrado y posgrado. Los resultados del estudio piloto arrojaron datos estadísticos tanto para pregrado como posgrado, como el promedio y la desviación estándar, necesarios para llegar al tamaño muestral. El valor mínimo de la muestra para hacer válido el presente estudio en pregrado fue de 148 y para posgrado 120 tratamientos de conductos radiculares.

Tomando en cuenta lo antes mencionado, fueron observadas 192 historias de pregrado y 136 historias de posgrado. Quedando al final 174 y 131 tratamientos de conductos para pregrado y posgrado respectivamente. Se excluyeron en total 23 historias clínicas: 7 de ellas 
presentaban tratamiento inconcluso, y el resto no especificaban datos ni en la hoja de evolución, ni evaluación presentes en la historia clínica.

Se realizó una evaluación descriptiva de la distribución de los tipos de diagnósticos pulpares y periapicales: tomando en cuenta la nueva clasificación y número de sesiones utilizadas en cada tratamiento de conducto radicular. Esta fue realizada considerando número de sesiones, diagnóstico pulpar vs número de sesiones y diagnóstico periapical vs número de sesiones, tanto en pregrado como en posgrado.

En la Tabla 1 se describe la frecuencia de sesiones que son requeridas para culminar un tratamiento de conducto. En el pregrado es igual de frecuente realizar tratamientos de conductos en una y dos sesiones
$(44,25 \%)$ y en posgrado es frecuente realizarlo en una sesión $(40,46 \%)$.

En la Tabla 2 se observa la frecuencia de los diferentes diagnósticos pulpares vs número de sesiones en Pregrado. Donde principalmente los diagnósticos de pulpitis irreversible asintomática son obturados en una sesión $(13,22 \%)$ y los diagnósticos de pulpitis irreversible sintomática y necrosis pulpar son obturados mayormente en dos sesiones (11,49\% y $15,52 \%$ respectivamente).

En la Tabla 3 se observa la frecuencia de los diagnósticos pulpares vs número de sesiones en posgrado. Los diagnósticos de pulpitis irreversible sintomática y necrosis pulpar fueron obturados en dos sesiones $(11,45 \%$ y $7,63 \%$ respectivamente) y los diagnósticos de pulpitis irreversible sintomática y tratamiento de conductos previo fueron obturados en una sesión $(8,4 \%)$.

En la Tabla 4 se observa la frecuencia de los diferentes diagnósticos periapicales vs número de sesiones en pregrado. Donde principalmente los diagnósticos de periodontitis apical asintomática fueron obturados tanto en una como en dos sesiones $(19,15 \%)$.

En la Tabla 5 se observa la frecuencia de los diferentes diagnósticos periapicales vs número de sesiones en Posgrado. Donde las piezas con diagnóstico periapical de periodonto sano, periodontitis apical sintomática y periodontitis apical asintomática fueron obturadas con mayor frecuencia en dos sesiones $(16 \%, 10 \%$ y $12 \%$ respectivamente).

Tabla 1. Número de sesiones para realizar tratamientos de conductos en pregrado y posgrado de Endodoncia.

\begin{tabular}{lcccc}
\hline & \multicolumn{2}{c}{ PREGRADO } & \multicolumn{2}{c}{ POSGRADO } \\
& $\mathrm{n}$ & $\%$ & $\mathrm{n}$ & $\%$ \\
\hline 1 Sesión & 77 & 44,25 & 53 & 40,46 \\
2 Sesiones & 77 & 44,25 & 43 & 32,82 \\
3 Sesiones & 17 & 9,77 & 14 & 10,69 \\
Más de 3 sesiones & 3 & 1,72 & 21 & 16,03 \\
\multicolumn{1}{c}{ TOTAL } & 174 & 100 & 131 & 100 \\
\hline
\end{tabular}

Tabla 2. Frecuencia de sesiones vs diagnóstico pulpar en pregrado.

\begin{tabular}{lccccccccccc}
\hline & \multicolumn{2}{c}{ 1 Sesión } & \multicolumn{2}{c}{ 2 Sesiones } & \multicolumn{3}{c}{ 3 Sesiones } & \multicolumn{2}{c}{ Más de 3 sesiones } & \multicolumn{2}{c}{ TOTAL } \\
& $\mathrm{n}$ & $\%$ & $\mathrm{n}$ & $\%$ & $\mathrm{n}$ & $\%$ & $\mathrm{n}$ & $\%$ & $\mathrm{n}$ & $\%$ \\
\hline Pulpa vital & 7 & 4,03 & 4 & 2,3 & 1 & 0,58 & 0 & 0 & 12 & 6,9 \\
Pulpitis reversible & 5 & 2,87 & 3 & 1,72 & 1 & 0,58 & 0 & 0 & 9 & 5,17 \\
Pulpitis irreversible sintomática & 18 & 10,34 & 20 & 11,49 & 5 & 2,87 & 0 & 0 & 43 & 24,71 \\
Pulpitis irreversible asintomática & 23 & 13,22 & 18 & 10,34 & 3 & 1,72 & 1 & 0,58 & 45 & 25,86 \\
Necrosis pulpar & 14 & 8,05 & 27 & 15,52 & 7 & 4,02 & 2 & 1,15 & 50 & 28,74 \\
Tratamiento de conductos previo & 9 & 5,17 & 4 & 2,29 & 0 & 0 & 0 & 0 & 13 & 7,47 \\
Tratamiento iniciado & 1 & 0,58 & 1 & 0,58 & 0 & 0 & 0 & 0 & 2 & 1,15 \\
TOTAL & & & & & & & & & 174 & 100 \\
\hline
\end{tabular}


Tabla 3. Frecuencia de sesiones vs diagnóstico pulpar en posgrado

\begin{tabular}{|c|c|c|c|c|c|c|c|c|c|c|}
\hline & \multicolumn{2}{|c|}{1 Sesión } & \multicolumn{2}{|c|}{2 Sesiones } & \multicolumn{2}{|c|}{3 Sesiones } & \multicolumn{2}{|c|}{3 o más Sesiones } & \multicolumn{2}{|c|}{ TOTAL } \\
\hline & $\mathrm{n}$ & $\%$ & $\mathrm{n}$ & $\%$ & $\mathrm{n}$ & $\%$ & $\mathrm{n}$ & $\%$ & $\mathrm{n}$ & $\%$ \\
\hline Pulpa vital & 10 & 7,64 & 1 & 0,76 & 0 & 0 & 0 & 0 & 11 & 8,4 \\
\hline Pulpitis reversible & 2 & 1,53 & 0 & 0 & 0 & 0 & 0 & 0 & 2 & 1,53 \\
\hline Pulpitis irreversible sintomática & 11 & 8,4 & 15 & 11,45 & 4 & 3,05 & 5 & 3,82 & 35 & 26,72 \\
\hline Pulpitis irreversible asintomática & 11 & 8,4 & 9 & 6,87 & 4 & 3,05 & 2 & 1,53 & 26 & 19,85 \\
\hline Necrosis pulpar & 8 & 6,11 & 10 & 7,63 & 2 & 1,53 & 6 & 4,58 & 26 & 19,85 \\
\hline Tratamiento de conductos previo & 11 & 8,4 & 7 & 5,34 & 4 & 3,05 & 8 & 6,11 & 30 & 22,9 \\
\hline Tratamiento iniciado & 0 & 0 & 1 & 0,76 & 0 & 0 & 0 & 0 & 1 & 0,76 \\
\hline TOTAL & & & & & & & & & 131 & 100 \\
\hline
\end{tabular}

Tabla 4. Frecuencia de sesiones vs diagnóstico periapical en pregrado.

\begin{tabular}{|c|c|c|c|c|c|c|c|c|c|c|}
\hline & \multicolumn{2}{|c|}{1 Sesión } & \multicolumn{2}{|c|}{2 Sesiones } & \multicolumn{2}{|c|}{3 Sesiones } & \multicolumn{2}{|c|}{3 o más Sesiones } & \multicolumn{2}{|c|}{ TOTAL } \\
\hline & $\mathrm{n}$ & $\%$ & $\mathrm{n}$ & $\%$ & $\mathrm{n}$ & $\%$ & $\mathrm{n}$ & $\%$ & $\mathrm{n}$ & $\%$ \\
\hline Tejido apical normal & 2 & 4,26 & 2 & 4,25 & 0 & 0 & 0 & 0 & 4 & 8,51 \\
\hline Periodontitis apical sintomática & 8 & 17,02 & 7 & 14,89 & 0 & 0 & 0 & 0 & 15 & 31,91 \\
\hline Periodontitis apical asintomática & 9 & 19,15 & 9 & 19,15 & 0 & 0 & 0 & 0 & 18 & 38,3 \\
\hline Absceso apical agudo & 0 & 0 & 1 & 2,13 & 1 & 2,13 & 0 & 0 & 2 & 4,25 \\
\hline Absceso apical crónico & 2 & 4,25 & 2 & 4,25 & 3 & 6,38 & 0 & 0 & 7 & 14,89 \\
\hline Osteitis condensante & 0 & 0 & 1 & 2,13 & 0 & 0 & 0 & 0 & 1 & 2,13 \\
\hline TOTAL & & & & & & & & & 47 & 100 \\
\hline
\end{tabular}

Tabla 5. Frecuencia de sesiones vs diagnóstico periapical en posgrado.

\begin{tabular}{|c|c|c|c|c|c|c|c|c|c|c|}
\hline & \multicolumn{2}{|c|}{1 Sesión } & \multicolumn{2}{|c|}{2 Sesiones } & \multicolumn{2}{|c|}{3 Sesiones } & \multicolumn{2}{|c|}{$\begin{array}{c}3 \text { o más Ses- } \\
\text { iones }\end{array}$} & \multicolumn{2}{|c|}{ TOTAL } \\
\hline & $\mathrm{n}$ & $\%$ & $\mathrm{n}$ & $\%$ & $\mathrm{n}$ & $\%$ & $\mathrm{n}$ & $\%$ & $\mathrm{n}$ & $\%$ \\
\hline Tejido apical normal & 7 & 14 & 8 & 16 & 1 & 2 & 3 & 6 & 19 & 38 \\
\hline $\begin{array}{l}\text { Periodontitis apical } \\
\text { sintomática }\end{array}$ & 2 & 4 & 5 & 10 & 1 & 2 & 4 & 8 & 12 & 24 \\
\hline $\begin{array}{l}\text { Periodontitis apical } \\
\text { asintomática }\end{array}$ & 3 & 6 & 6 & 12 & 1 & 2 & 5 & 10 & 15 & 30 \\
\hline Absceso apical agudo & 0 & 0 & 0 & 0 & 1 & 2 & 0 & 0 & 1 & 2 \\
\hline Absceso apical crónico & 0 & 0 & 2 & 4 & 1 & 2 & 0 & 0 & 3 & 6 \\
\hline Osteitis condensante & 0 & 0 & 0 & 0 & 0 & 0 & 0 & 0 & 0 & 0 \\
\hline TOTAL & & & & & & & & & 50 & 100 \\
\hline
\end{tabular}




\section{Discusión}

En posgrado, lo más frecuente fue realizar tratamientos de conductos en 1 sesión $(40,46 \%)$ (Tabla $1)$; este resultado se puede explicar debido a la mayor destreza y entrenamiento continuo que tienen los estudiantes. Además, utilizan técnicas de instrumentación rotatoria y obturación termoplástica; lo que posiblemente disminuye el tiempo operatorio. Vaudt et al. reportaron que el tiempo de preparación biomecánica utilizando sistemas rotatorios disminuía significativamente comparado con el tiempo requerido en la instrumentación manual (14). Sánchez et al. mencionan que el uso de instrumentos rotatorios de níquel titanio ha revolucionado la endodoncia, disminuyendo el tiempo de trabajo y la fatiga del operador; además de facilitar la conformación del sistema de conductos radiculares sin alteración de la anatomía del mismo (15).

El número de sesiones para realizar tratamientos de conductos en pregrado es igual de frecuente en uno o en dos sesiones (44,25\%) (Tabla 1). Dichos tratamientos se rigen según el esquema de trabajo propuesto por la sección de Endodoncia de la FE-UPCH. Se sabe que los estudiantes de pregrado están en proceso de aprendizaje de las técnicas de trabajo, añadiendo su inexperiencia relativa; se podría pensar que el seguimiento de los casos no es muy adecuado o que el registro de las actividades diarias por parte de los estudiantes no está siendo correctamente supervisada. Aunque no fue motivo de la investigación, una posible explicación de encontrar tratamientos de conductos en pregrado con igual frecuencia en una o dos sesiones, es probablemente debido a que mucho de los estudiantes de pregrado suelen utilizar mayor tiempo clínico por sesión que los estudiantes de posgrado, pudiendo emplear casi 4 horas por sesión, lo cual influye en realizar los tratamientos en menos sesiones.

Además podríamos considerar que en el caso de pregrado se presentan casos más sencillos de tratar y es posible culminar el tratamiento de conductos en menos sesiones. El hecho que sean pacientes buscados por el propio operador hace que sea menos probable la posibilidad de encontrar un paciente con sintomatología dolorosa exagerada, lo que disminuye, posiblemente las sesiones en las que la pieza dentaria pueda ser tratada.

El resultado más específico indica que en posgrado se realizan tratamientos de conductos en más de 3 sesiones (16,03\%); debido a que en este servicio son derivadas piezas con anatomía compleja y mayor curvatura, lo que dificultaría a los estudiantes de posgrado realizar el tratamiento en menos sesiones. Leonardo (2005), menciona que es necesario tener en cuenta que los distintos diagnósticos pulpares, con o sin lesión periapical crónica visible radiográficamente son entidades patológicas diferentes, y por lo tanto necesitan procedimientos diferentes, que podrían abarcar más de una sesión en la terapia endodóntica. (4) En general, la mayor dificultad en el tratamiento de conductos, desde todo punto de vista, suelen ser las piezas molares. Ashkenaz señala que además de las dificultades anatómicas y la accesibilidad de los dientes multirradiculares, la tolerancia del paciente hacia un tratamiento prolongado pueda ser limitada (16). Gartner sugiere tener en cuenta que un diente más posterior genera menor visibilidad y acceso, dificultando el tratamiento en una sesión. Y si aumentamos la condición de tener un diente más angulado, este irá en desmedro de su accesibilidad, aumentando también el número de sesiones (9). Van Nieuwenhuysen et al. realizaron un estudio en el que observaron que los dientes más frecuentemente tratados fueron los molares mandibulares particularmente la primera molar y que los incisivos maxilares fueron retratados 20 veces más frecuentemente que los incisivos mandibulares (17). Reátegui encontró relación en cuanto a la distribución de las piezas dentarias retratadas según la arcada dentaria. Encontró que en el maxilar superior las más frecuentes fueron incisivos con 24 casos $(42 \%)$ y en el maxilar inferior fueron las molares con $37 \mathrm{ca}$ $\operatorname{sos}(82 \%)(18)$.

Las piezas con diagnóstico de pulpitis irreversible sintomática tanto en pregrado como en posgrado de la FE-UPCH $(24,71 \%$ y $26,72 \%$ respectivamente) (Tablas 2 y 3 ), fueron tratadas mayormente en 2 sesiones ( $11.49 \%$ en el pregrado y $11.45 \%$ en el posgrado). Mientras que las piezas con diagnóstico de pulpitis irreversible asintomática fueron tratadas en su mayoría en 1 sesión $(13,22 \%$ en pregrado y $8.4 \%$ en posgrado). Esto puede deberse a la consideración de los operadores con respecto a la sintomatología previa de la pieza.

Aunque se debe considerar que cuando el dolor está presente y se tiene un diagnóstico pulpitis irreversible (ya sea asintomática o sintomática), la obturación se puede hacer en la visita inicial, puesto que la eliminación del tejido con vitali- 
dad resolverá en general la molestia del paciente (19).

Los casos de piezas dentarias con necrosis pulpar en pregrado y posgrado de la FE-UPCH fueron resueltos mayormente en 2 sesiones $(35,06 \%$ y $23,25 \%$ respectivamente). Una creencia fuertemente arraigada en varios clínicos, es que la endodoncia en una sesión aumenta el índice de exacerbaciones, las cuales suelen presentarse en dientes necróticos con lesión apical así como en retratamientos. Por ello para estos casos, aconsejan una terapia de varias sesiones con medicación de hidróxido de calcio (4), siendo retrasada la obturación hasta que los síntomas desaparezcan (20).

Debido al alto porcentaje de datos no registrados respecto al diagnóstico periapical (Tablas 4 y 5), tanto en pregrado $(72,99 \%)$, como en posgrado $(61,83 \%)$ de la FE-UPCH, no se podría determinar la tendencia de los operadores respecto al número de sesiones relacionándolas con esta variable. El conocimiento del estado pulpar y de los tejidos adyacentes es imprescindible para elaborar y plantear un adecuado plan de tratamiento. Con toda la información recopilada y en base a un fundamento científico, es necesario considerar que no hay patrones de tratamiento establecidos para cada pieza dentaria, aunque hablemos del mismo diagnóstico pulpar y periapical.

\section{Conclusiones}

1. En pregrado de la FEUPCH es igual de frecuente realizar tratamientos de conductos en una o dos sesiones (44,25\%). Mientras que en postgrado, los tratamientos de conductos se concluyen en una sesión $(40.46 \%)$.
2. En pregrado de la FE$\mathrm{UPCH}$, el diagnóstico pulpar más frecuente fue la necrosis pulpar $(28,73 \%)$ siendo tratada en dos sesiones $(15,52 \%)$.

3. En el Servicio de Endodoncia de Postgrado de la FE-UPCH, el diagnóstico pulpar más frecuente fue la pulpitis irreversible sintomática $(26.71 \%)$ siendo tratada en dos sesiones $(11,42 \%)$.

4. El diagnóstico periapical en pregrado y postgrado de la FE$\mathrm{UPCH}$, no fue registrado en $72,99 \%$ $y$ en $61,83 \%$ respectivamente.

\section{Referencias Bibliográficas.}

1. Alves R, Nogueira E. Endodontia. Trauma. 2da ed. Sao Paulo: Artes médicas; 2002.

2. Trallero T. Single Visit endodontics: A clinical study. J Endod. 1983; 9(4): 147-52.

3. Baumann M, Beer R. Endodoncia. 2da ed. Madrid: Elsevier; 2008.

4. Leonardo M, Leal J. Endodoncia: Tratamiento de conductos radiculares. $2^{\circ}$ ed. Buenos Aires: Médica Panamericana, 1994.

5. Morales G. Tratamientos conservadores de la vitalidad pulpar y tratamiento endodóntico en una sesión. Tesis de Bachiller. Lima, Perú. Universidad Nacional Mayor de San Marcos, 2000.

6. Malhotra N, Kundabala M, Acharya S. Contemporary endodontic approach: single-visit root canal treatment revisited. Endodontic Practice Today. 2009; 3(3): 215-25.

7. Sathonrn C, Parashos P, Messer $H$. Australian endodontists perceptions of single and multiple visit root canal treatment. Int Endod J. 2009; 42(9): 811-8.

8. Mubarak A, Abu-Bark N, Ibra- him Y. Postoperative pain in multiple-visit and single-visit root canal treatment. J Endod. 2010; 36(1): 36-9.

9. Quiterio N. Tratamiento endodóntico en una sesión (Internet). Caracas, Venezuela: Carlos Boveda; 1999. (Citado en noviembre del 1999) Disponible en: http://www.carlosboveda.com/ Odontologosfolder/odontoinvitadoold/odontoinvitado4.htm

10. Soares I, Goldberg F. Endodoncia: Técnica y fundamentos. 1ra ed. Buenos Aires: Médica Panamericana; 2002.

11. Peters L, Van Winkelhoff A-J, Buijs J, Wesselink P. Effects of instrumentation, irrigation and dressing with calcium hydroxide on infection in pulpless teeth with periapical bone lesions. Int Endod J. 2002; 35(1): 13-21.

12.Pinheiro ET, Gomes BPFA, Ferraz CCR, Sousa ELR, Teixeira FB, Souza-Filho FJ. Microorganisms from canals of root-filled teeth with periapical lesions. Int Endod J. 2003; 36(1): 1-11.

13. Peters O, Paqué F. Current developments in rotary root canal instrument technology and clinical use: A review. Quintessence Int. 2010; 41(6): 479-88.

14. Vaudt J, Bitter K, Kielbassa A. Evaluation of rotary root canal instruments in vitro: a review. ENDO. 2007; 1(3):189-203.

15.Sánchez J, Garzón J, Martínez J, Villavicencio J, Cárdenas R. Estudio comparativo del trabajo biomecánico del sistema Protaper y la instrumentación manual in vitro. Rev ADM. 2008; 65(3): 126-32.

16. Ashkenaz PJ. One visit endodontics. Dent Clin North Am. 1984; 28: 853-63.

17. Van Nieuwenhuysen JP, Aouar M, D'Hoore W. Retreatment or 
radiographic monotoring in endodontics. Int Endod J. 1994; 27(2): 75-81.

18. Reátegui P. Frecuencia de retratamientos endodónticos según grupo dentario de pacientes que acudieron al servicio de Postgrado de Endodoncia de la Clínica Estomatológica Central
Cayetano Heredia durante el periodo Enero 1995 a Enero 1997. Tesis de Bachiller. Lima, Perú. Universidad Peruana Cayetano Heredia, 1998.

19. Gutman J, Dumsha T, Lovdahl P. Soluciones en problemas en endodoncia, prevención, identificación y tratamiento. 5ta ed.
Madrid: Elsevier; 2007.

20. Villena H. Terapia pulpar. 2da ed. Lima: Ripano. 2012.

Recibido : 12-10-2011

Aceptado: 20-01-2012 